\title{
PIELOLITOTOMÍA LAPAROSCÓPICA EN RIÑÓN PELVIANO
}

\author{
L.A. FARIÑA PÉREZ, J. CAMBRONERO SANTOS, F. MEIJIDE RICO, E.R. ZUNGRI TELO \\ Servicio de Urología. Centro Médico POVISA. Vigo. Pontevedra. \\ Actas Urol Esp. 28 (8): 620-623, 2004
}

\section{RESUMEN}

PIELOLITOTOMÍA LAPAROSCÓPICA EN RIÑÓN PELVIANO

INTRODUCCIÓN: El riñón pelviano afecto de cálculos es un caso particularmente raro de litiasis compleja. Diversos procedimientos, percutáneos y laparoscópicos, se han propuesto en los últimos años para resolverlo de manera poco invasiva.

CASO CLÍNICO: Se muestra el procedimiento de pielolitotomía laparoscópica mediante tres únicos accesos, en un varón con cálculos sintomáticos en un riñón pelviano izquierdo con función normal. Se extrajeron dos cálculos con ayuda del nefroscopio flexible introducido a través de uno de los accesos, y cerrando la pelvis renal con sutura intracorpórea.

COMENTARIO: La pielolitotomía laparoscópica de un riñón pelviano es poco invasiva y puede hacerse con facilidad, haciendo innecesaria una laparotomía. La técnica laparoscópica debe tenerse en cuenta en el infrecuente paciente con litiasis compleja en el que se considere una intervención abierta. Este es el segundo caso de riñón pelviano operado mediante laparoscopia, en la bibliografía española.

PALABRAS CLAVE: Riñón pelviano. Litiasis. Pielolitotomía. Laparoscopia.

\section{ABSTRACT}

\section{LAPAROSCOPIC PYELOLITHOTOMY IN A PELVIC KIDNEY}

INTRODUCTION: Pelvic renal ectopia bearing stones is a particularly rare case of complex calculi. Several percutaneous and laparoscopic procedures had been proposed in the last years to treat this challenge problem in a minimally invasive way.

CLINICAL CASE: We describe the procedure of laparoscopic pyelolithotomy under general anesthesia, using three port sites, in a male patient with symptomatic stones in a left pelvic kidney with normal function. Two stones were successfully removed, with the help of flexible nephroscopy through a port, and the pyelotomy was closed with intracorporeal suture.

COMMENT: Laparoscopic pyelolithotomy of a pelvic kidney is a minimally invasive technique that is not difficult to perform, making laparotomy unnecessary. Laparoscopic technique must be take into account when dealing with the infrequent complex stone patient candidate to an open stone surgery. A review of the Spanish bibliography shows this to be the second reported case of pelvic kidney calculi treated by laparoscopic pyelolithotomy.

KEY WORDS: Pelvic kidney. Calculi. Pyelolithotomy. Laparoscopy. 
$\mathrm{E}^{1}$ riñón ectópico pelviano se caracteriza por una vascularización anómala y un sistema colector rotado, en posición variable. Su forma, posición y orientación insólitas pueden condicionar un drenaje inadecuado que lo haría más propenso a la obstrucción y a tener cálculos, los cuales tradicionalmente han sido tratados mediante cirugía abierta. El tratamiento con litotricia extracorpórea por ondas de choque, está limitado por la presencia de las estructuras óseas y las asas intestinales vecinas, y de los genitales internos en la mujer, que disminuyen la eficacia de las ondas o aumentan el riesgo de complicaciones indeseables ${ }^{1}$. Diversos procedimientos, percutáneos y laparoscópicos, se han propuesto en los últimos años para tratar de manera poco invasiva la litiasis del riñón pelviano. Describimos el procedimiento de pielolitotomía laparoscópica mediante tres únicos accesos, en un varón con cálculos sintomáticos en un riñón pelviano izquierdo con función normal.

\section{CASO CLÍNICO}

Paciente que consultó por dolor suprapúbico agudo con hematuria leve, sin otros síntomas miccionales. El estudio urológico consecuente mostró un riñón pelviano izquierdo, con la vía urinaria anterior y dos cálculos pielo-caliciales de $12 \mathrm{~mm}$ de diámetro mayor (Fig. 1). La radioscopia no permitió identificar los cálculos con seguridad, haciendo imposible la litotricia extracorpórea por ondas de choque, y el paciente prefirió retrasar otras opciones de tratamiento. Sin embargo en los meses siguientes sufrió cuadros repetidos de dolor intenso que finalmente le impedian hacer su trabajo como transportista. Dado que el estudio topográfico mostraba un riñón con la vía urinaria anterior y cálculos más accesibles por esta vía, se optó por una extracción laparoscópica de los cálculos.

Con el paciente bajo anestesia general se implantó un catéter ureteral en el lado izquierdo. Se colocaron tres únicos accesos laparoscópicos, en región umbilical (óptica) y ambas fosas iliacas.

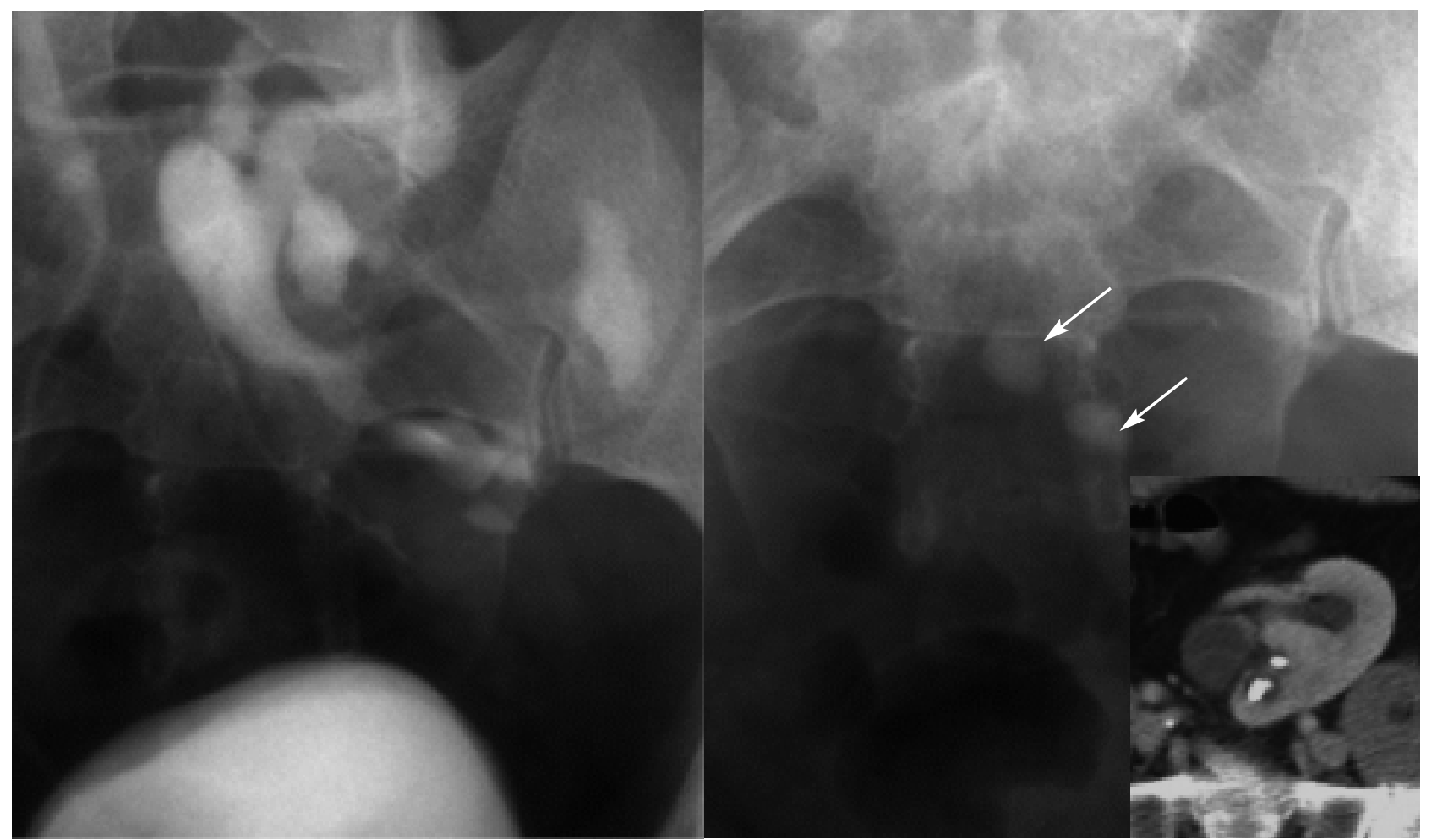

FIGURA 1. Radiografía simple de abdomen y urografía endovenosa mostrando 2 cálculos pielo-caliciales en un riñón pelviano. Abajo, insertado, TC sin contraste muestra la orientación anterior de la pelvis renal y su relación con intestino, vasos y sacro. 
Se estableció un neumoperitoneo estándar y con una posición de Trendelenburg moderada, separando algunas asas intestinales, se identificó enseguida la prominencia correspondiente al riñón pelviano. La administración por el catéter ureteral de un colorante, permitió localizar la pelvis renal, que fue abierta con endotijera en unos $1,5 \mathrm{~cm}$ sin necesidad de coagular o clipar vasos renales. No fue necesario usar la radioscopia. Uno de los cálculos inmediatamente a la vista se extrajo con endopinza. Se introdujo el nefroscopio flexible por el trocar derecho y bajo control laparoscópico se dirigió a la pelvis renal, para explorar la vía urinaria: el segundo cálculo se localizó en el cáliz inferior y fue extraído con la pinza del nefroscopio. La pelvis se cerró con tres puntos de sutura intracorpórea (Fig. 2). El tiempo operatorio fue de 100 minutos. El dolor postoperatorio se trató con analgésicos menores, el catéter se retiró al tercer día y los estudios de imagen post-operatorios mostraron una función íntegra. Los cálculos fueron de oxalato cálcico y el estudio metabólico mostró hiperuricosuria leve.

\section{COMENTARIO}

Eshghi et al. ${ }^{2,3}$ fueron los primeros en proponer la nefrolitotomía percutánea con asistencia laparoscópica para un riñón pelviano con cálculos, que iniciaban con una punción retrógada del cáliz renal y continuaban con dilatación anterógrada del tracto y la litotricia percutánea, bajo visión laparoscópica simultánea de las asas intestinales, para evitar su lesión. Confirmando que era reproducible, esta técnica fue imitada por otros, con algunas variantes: en estos casos sucesivos se vio que la punción anterógrada de la vía urinaria era más idónea, que el laparoscopio era sobre todo útil para separar las asas intestinales antes de la punción ${ }^{4,5}$ y que era también posible hacerla con una insuflación extraperitoneal ${ }^{6}$.

Desai y Jasani ${ }^{7}$ propusieron evitar la ayuda laparoscópica al publicar su experiencia de nefrolitotomía del riñón pelviano con una técnica percutánea pura en nueve pacientes, auxiliándose con una lordosis forzada y control ecográfico de la punción para evitar la lesión intestinal, y usando un acceso a la vía urinaria

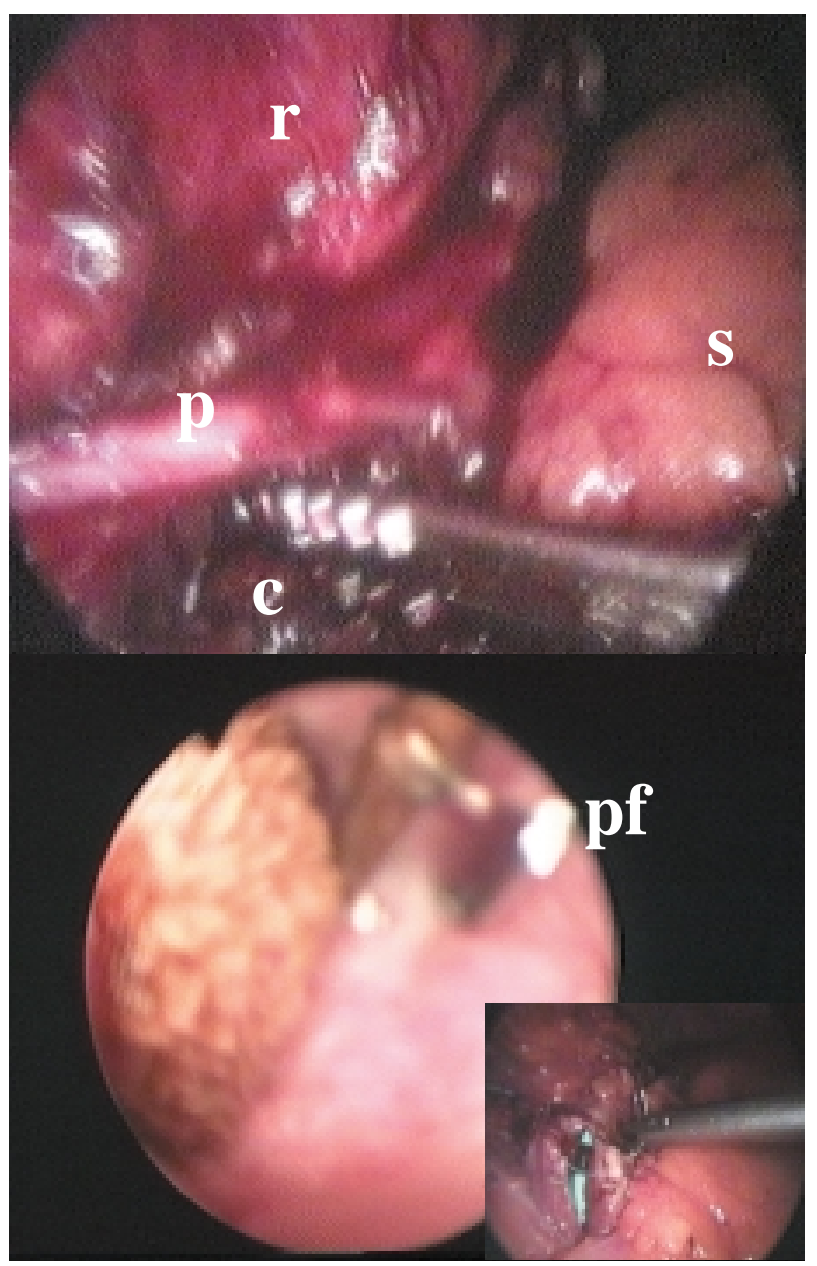

FIGURA 2. Arriba, extracción con pinza laparoscópica de un cálculo (c: cálculo, p: pelvis renal, r: riñón, s: sigma). Abajo, imagen a través de nefroscopio flexible extrayendo un segundo cálculo (pf: pinza flexible). Insertado, sutura endoscópica de la pelvis renal.

madurada durante algunos días en un tercio de los pacientes, que así eran operados en dos tiempos. Afirman que el desplazamiento de las asas con ayuda de la sonda ecográfica es segura, pero debido a la variable posición y configuración anatómica de estos riñones y su anómala circulación, sus observaciones no pueden generalizarse.

La alternativa de la nefrolitotomía percutánea por un acceso posterior, transglúteo, a través de la escotadura ciática mayor, fue planificada con el estudio de tomografía computarizada, y realizada con éxito por Watterson et al. ${ }^{8}$ en una mujer joven y delgada con un cálculo calicial inferior de un riñón situado en la pelvis 
posterior. Este acceso parece conveniente en algunos casos, pero la longitud de los instrumentos endoscópicos disponibles lo contraindicaría en pacientes gruesos o en riñones situados en la pelvis anterior, más distantes de la piel. En el caso de Monga et al. $^{9}$ un acceso posterior y medial a través de la musculatura paraespinal en una mujer joven se complicó con una neuropatía femoral grave.

Realizada por primera vez por Harmon et al. en $1996^{10}$ y por Hoenig et al. en $1997^{11}$, la extracción de cálculos del riñón pélvico mediante uso exclusivo de instrumentos laparoscópicos, fue la opción que nosotros elegimos. El uso del nefroscopio flexible permitió hacer una muy limitada incisión piélica, disminuyendo el tiempo de reparación y el riesgo de fístula. El examen de la vía intrarrenal mediante el nefroscopio flexible evita la revisión en un segundo tiempo recomendada por otros autores, ya que en caso de cálculos múltiples o de cálculos grandes que necesiten fragmentación intracorpórea, es difícil asegurar radiológicamente la limpieza, debido a las estructuras óseas adyacentes. Este es el segundo riñón pelviano operado mediante laparoscopia, en la bibliografía española, después del informado por Valdivia et al. ${ }^{12}$ en el año 2002.

En conclusión, la pielolitotomía laparoscópica de un riñón pelviano es poco invasiva y puede hacerse con facilidad, haciendo innecesaria una laparotomía. Las posibilidades que ofrece la laparoscopia para complementar el tratamiento poco invasivo de litiasis complejas, han disminuido aún más las raras indicaciones que quedaban de tratamiento quirúrgico clásico de los cálculos, por dificultades de la cirugía percutánea o de la litotricia extracorpórea-, independientemente de su localización o de la presencia de variantes anatómicas $^{13}$.

\section{REFERENCIAS}

1. Lingeman JE, Smith LH, Woods JR, Newman DM. Urinary calculi. ESWL, Endourology and Medical Therapy. Lea \& Febiger, Filadelfia 1989:223-229.

2. Eshghi AM, Roth JS, Smith AD. Percutaneous transperitoneal approach to a pelvic kidney for endourological removal of staghorn calculus. J Urol 1985;134:525-527.

3. Lee CK, Smith AD. Percutaneous transperitoneal approach to the pelvic kidney for endourologic removal of calculus. Three cases with two successes. J Endourol 1992;6:133-135.

4. Toth C, Holman E, Pásztor I, Khan AM. Laparoscopically controlled and assisted percutaneous transperitoneal nephrolithotomy in a pelvic dystopic kidney. J Endourol 1993; 7:303305.

5. Holman E, Toth C. Laparoscopically assisted percutaneous transperitoneal nephrolithotomy in pelvic dystopic kidneys: experience in 15 successful cases. J Laparoendosc Adv Surg Tech A 1998;8:431-435.

6. Troxel SA, Low RK, Das S. Extraperitoneal laparoscopyassisted percutaneous nephrolithotomy in a left pelvic kidney. J Endourol 2002;16:655-657.

7. Desai MR, Jasani A. Percutaneous nephrolithotripsy in ectopic kidneys. J Endourol 2000;14:289-292.

8. Watterson JD, Cook A, Sahajpal R, Bennett J, Denstedt JD. Percutaneous nephrolithotomy of a pelvic kidney: a posterior approach through the greater sciatic foramen. J Urol 2001; 166:209-210.

9. Monga M, Castañeda WR, Thomas R. Femoral neuropathy following percutaneous nephrolithotomy of a pelvic kidney. Urology 1995;45:1059-1061.

10. Harmon WJ, Kleer E, Segura JW. Laparoscopic pyelolithotomy for calculus removal in a pelvic kidney. J Urol 1996;155: 2019-2020.

11. Hoenig DM, Shalhav AL, Elbahnasy AM, Mcdougall EM, Clayman RV. Laparoscopic pyelolithotomy in a pelvic kidney: a case report and review of the literature. J Soc Laparoendosc Surg 1997;1:163-165.

12. Valdivia JG, Blasco B, Elizalde A et al. Nuevas fronteras en endourologia: endoscopia intra y extraluminal. Actas Urol Esp 2002;26(Supl); resumen V19.

13. Ramakumar S, Segura JW. Laparoscopic surgery for renal urolithiasis: pyelolithotomy, caliceal diverticulectomy and treatment of stones in a pelvic kidney. J Endourol 2000; 14:829-832.

Dr. L.A. Fariña

Servicio de Urología. Centro Médico POVISA

C/ Salamanca, 5

36211 Vigo (Pontevedra)

(Trabajo recibido el 6 noviembre de 2003) 